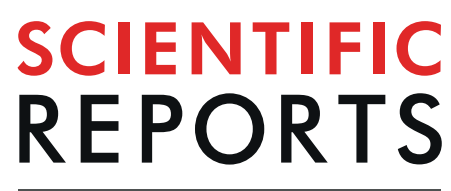

natureresearch

\title{
Selective autophagy, lipophagy and mitophagy, in the Harderian gland along the oestrous cycle: a potential retrieval effect of melatonin
}

\author{
Marina García-Macia $\mathbb{1}^{1,6,7 *}$, Adrián Santos-Ledo ${ }^{2,7}$, Beatriz Caballero ${ }^{3}$, \\ Adrian Rubio-González ${ }^{3}$, Beatriz de Luxán-Delgado ${ }^{3,5}$, Yaiza Potes ${ }^{3}$, \\ Susana $\mathrm{M}^{\mathrm{a}}$. Rodríguez-González ${ }^{3}$, José Antonio Boga ${ }^{4}$ \& Ana Coto-Montes $\mathbb{1}^{3 *}$
}

Sexual dimorphism has been reported in many processes. However, sexual bias in favour of the use of males is very present in science. One of the main reasons is that the impact of hormones in diverse pathways and processes such as autophagy have not been properly addressed in vivo. The Harderian gland is a perfect model to study autophagic modulation as it exhibits important changes during the oestrous cycle. The aim of this study is to identify the main processes behind Harderian gland differences under oestrous cycle and their modulator. In the present study we show that redox-sensitive transcription factors have an essential role: NF- $\kappa$ B may activate SOSTM1/p62 in oestrus, promoting selective types of autophagy: mitophagy and lipophagy. Nrf2 activation in dioestrus, leads the retrieval phase and restoration of mitochondrial homeostasis. Melatonin's receptors show higher expression in dioestrus, leading to decreases in pro-inflammatory mediators and enhanced Nrf2 expression. Consequently, autophagy is blocked, and porphyrin release is reduced. All these results point to melatonin as one of the main modulators of the changes in autophagy during the oestrous cycle.

Sex bias is still a clear issue in biomedical research, although sexual dimorphism has been described in many cellular processes under pathological and physiological conditions ${ }^{1,2}$. Sex-dependent differences in the activation of autophagy have been reported mainly in vitro ${ }^{3,4}$. It has been reported that hormonal changes stimulate autophagy in mammary gland, but just using in vitro models ${ }^{5,6}$. Androgens also modulate autophagy in flank organ, which is a sebaceous gland, in vivo ${ }^{7}$. However, how autophagy is modulated under sexual hormone variations, as during the oestrous cycle, is poorly understood.

The Syrian hamster Harderian gland (HG) is a tubule-alveolar orbital gland formed by two main types of cells (type I and II) that secretes lipids favouring lubrication of the cornea ${ }^{8}$. The HG has other functions including the production of pheromones ${ }^{9}$, functions related to the pineal-gonadal axis ${ }^{10}$, and synthesis of indolamines ${ }^{11}$. HG exhibits marked sexual differences in relation to cell type and porphyrin production. In previous studies, we have demonstrated alterations of female gland activity during the oestrous cycle ${ }^{12}$. Interestingly, the oestrus phase presents the highest porphyrin-production activity, which has been associated with a prominent oxidative

${ }^{1}$ Institute of Cellular Medicine, Newcastle University, William Leech Building, NE2 4HH, Newcastle Upon Tyne, UK. ${ }^{2}$ Institute of Genetic Medicine, Newcastle University, International Centre for Life Central Parkway, NE1 3BZ, Newcastle Upon Tyne, UK. ${ }^{3}$ Departamento de Morfología y Biología Celular, Área de Biología Celular, Facultad de Medicina, Universidad de Oviedo, Julián Clavería s/n, 33006, Oviedo, Spain. ${ }^{4}$ Servicio de Microbiología, Hospital Universitario Central de Asturias, Avenida de Roma s/n., 33011, Oviedo, Spain. ${ }^{5}$ Present address: Barts Cancer Institute-Queen Mary, University of London, Centre for Tumour biology, John Vane Science Centre, Charterhouse Square, London, EC1M 6BO, UK. 'Present address: Instituto de Investigación Biomédica de Salamanca (IBSAL), Neuroenergetics and Metabolism Group, Institute of Functional Biology and Genomics, University of SalamancaCSIC, Zacarias Gonzalez, 2, 37007, Salamanca, Spain. ${ }^{7}$ These authors contributed equally: Marina García-Macia and Adrián Santos-Ledo. *email: marinagarciamacia@gmail.com; acoto@uniovi.es 
damage to proteins, together with a lower total antioxidant activity and higher autophagy, compared to the dioestrus phas $\mathrm{e}^{4,12}$. By contrast, the dioestrus phase shows a lower porphyrins production causing reduced oxidative stress levels, a decrease in proteolytic activities and a blockage of autophagy due to activation of $\mathrm{mTOR}^{12}$. Morphological changes also occur during the oestrous cycle, the Type II cells of the Harderian gland are more abundant during oestrus ${ }^{12}$.

The main difference between Type I and II cells is the size of the lipid droplets (LD). Type II cells present bigger and more abundant $\mathrm{LDs}^{13}$, suggesting that the LDs are active organelles in the HG. Previously, it has been reported that autophagy controls the mobilization of LDs to lysosomes ${ }^{14,15}$. This new type of LD degradation is called lipophagy ${ }^{14,15}$. Although it was first discovered in liver, it has also been observed in several cell types, where it acts as an energy source with the peculiar capability to provide large amounts of free fatty acids in a short time $^{14,15}$. Turnover of endogenous lipid stores is essentially developed by Lysosomal acid lipase (LAL) ${ }^{16,17}$, which could be considered an essential lipophagy marker. However, the presence of lipophagy in HG is still unconfirmed, even though lipid droplets are indispensable components of glandular cells.

The pineal melatonin is the endogenous synchronizer of the circadian rhythms in organisms, mainly of those related to the control of seasonal reproductive phenomena ${ }^{18}$. Likewise, melatonin and its metabolites are well-known antioxidants ${ }^{19}$. Moreover, melatonin is able to maintain mitochondrial homeostasis ${ }^{20,21}$, as it increases the activity of the mitochondrial respiratory complexes I and IV, which result in increased ATP production ${ }^{22}$. Melatonin plays and important role modulating the morphology and physiology of the $\mathrm{HG}^{11,23}$. Melatonin secretion depends on the phase of the oestrous cycle ${ }^{24}$ and it is diminished by gonadal steroid hormones ${ }^{25}$.

Based on these findings and considering the observed oscillations in autophagy activity as well as Type II cell abundance along the oestrous cycle ${ }^{12}$, the aim of this study was to identify the main modulators behind autophagic changes in the oestrous cycle and the involvement of the lipophagy.

\section{Results}

Melatonin receptors expression: cellular response to oxidative stress. The role of melatonin in oestrous cycle physiology can be assessed by the expression of melatonin receptors, which have been described in $\mathrm{HG}^{26} . \mathrm{MT}_{1}$ is a $\mathrm{G}$ protein-coupled receptor at the plasma membrane, and it is activated by the pineal-synthesized melatonin ${ }^{26}$. ROR $\alpha$ is a nuclear melatonin receptor that plays an important role in cell protection against oxidative stress ${ }^{27}$. Western-blot analyses for $\mathrm{MT}_{1}$ and $\mathrm{ROR} \alpha$ proteins showed higher expression levels of both in dioestrus (Fig. 1a, $\mathrm{p}<0.05$ ).

$\mathrm{Nrf2}$ is an essential transcription factor in the cellular response to oxidative stress ${ }^{28}$. Under basal conditions, nuclear levels of Nrf2 remain relatively low because it is sequestered in the cytosol ${ }^{19,28}$. High oxidative stress levels activate the canonical Nrf2 pathway ${ }^{29}$. Our study reveals a strong Nrf2 activation in the dioestrus phase, compared to the one in oestrus phase. Nrf2 showed higher expression both in total homogenates (Fig. $1 \mathrm{~b}, \mathrm{p}<0.05)$ and in nuclear fractions (Fig. 1d). To further analyse Nfr2 activation, Glucose-6Phosphate Dehydrogenase (Glucose$6 \mathrm{PDH}$ ) and SOD2 levels were tested. Both proteins also showed higher expression in dioestrus (Fig. 1f, $\mathrm{p}<0.01$ ).

Nuclear factor-kappa B (NF- $\kappa \mathrm{B})$ is a transcription factor that also plays a key role in cell responses to oxidative stress $^{30}$. We examined its activation state using immunoblot analysis of key proteins in the NF- $\kappa$ B pathway: 065 and phospho-p65 (Ser536). Activation occurs via phosphorylation of p65 at Ser536, and only the phosphorylated protein has nuclear localization and transcriptional activity ${ }^{4}$. Total amount of p 65 did not show significant differences between phases of the oestrous cycle of HG (Fig. 1b). However, phosphorylated p65 expression was significantly lower in dioestrus than oestrus phase (Fig. 1b). Thus, to characterize the NF- $\kappa B$ activation, we evaluated the ratio of phospho-p65 respect to total p65 protein and confirmed a lower level of phospho-p65/p65 in dioestrus compared to oestrus (Fig. $1 \mathrm{~b}, \mathrm{p}<0.001$ ). Oestrus and dioestrus nuclear fractions were evaluated to test phopho-p65 translocation to the nucleus, lower expression of phopho-p65 was found in dioestrus (Fig. 1c). Then, downstream proteins were evaluated: iNOS, COX2 and IL1 $\beta$, being last 2 proteins also pro inflammatory enzymes. All of them showed lower expression in the dioestrus phase (Fig. $1 \mathrm{e}, \mathrm{p}<0.05$ ). Our data suggest a lower activation level of the NF- $\kappa B$ pathway in the dioestrus phase.

In order to further understand the impact of NF- $\mathrm{B}$ on autophagy, $\mathrm{p} 50^{\mathrm{KO}}\left(\mathrm{NF}-\kappa \mathrm{B} 1^{\mathrm{KO}}\right)$ and wild type $(\mathrm{WT})$ mouse embryonic fibroblasts (MEFs) were used and p62 expression was examined. Mutant cells showed a reduction of p62 expression (Fig. S1). Furthermore, proteins degraded by selective autophagy: SDHB or complex II for mitophagy and Plin 2 and Plin 3 for lipophagy, were actively degraded in WT cells while accumulated in p50 ${ }^{\mathrm{KO}}$ ones. These results would indicate that deficient NF- $\kappa B$ signalling have a negative impact on autophagy.

Functional mitochondrial status. Mitochondria play an essential role in cell survival ${ }^{31}$, but their activity generates free radicals that alter the oxidative balance ${ }^{32}$. Mitochondria also play a key role in lipid metabolism ${ }^{33}$. To characterize mitochondrial functional status, we studied citrate synthase activity and ATP generation. Citrate synthase is a mitochondrial matrix enzyme, essential in Krebs cycle that is an indicator of healthy mitochondrial population $^{34}$. Based on this mitochondrial marker, the number of mitochondria is higher in dioestrus than in oestrus (Fig. 2a, p <0.05). Accordingly, ATP levels were also higher in dioestrus, indicating better mitochondrial efficiency (Fig. 2b, p < 0.001).

Mitophagy, the selective autophagy pathway to degrade defective mitochondria, was also assessed. The mitochondrial protein NIX has been described as an autophagy receptor, mediating the clearance of damaged mitochondria ${ }^{35}$. NIX expression was lower in dioestrus than oestrus (Fig. $2 c, p<0.01$ ). Using electron microscopy (EM) we corroborated the elevated presence of mitophagosomes during oestrus (Fig. 2d). Mitochondria are healthier in dioestrus, hence, their selective degradation through autophagy is less prominent during this phase.

Lipophagy. The LC3-interacting protein SQSTM1/p62 is a key autophagic protein for cell homeostasis mediating the selective specific degradation of protein aggregates and cytoplasmic bodies ${ }^{36}$. Recent studies have 
a
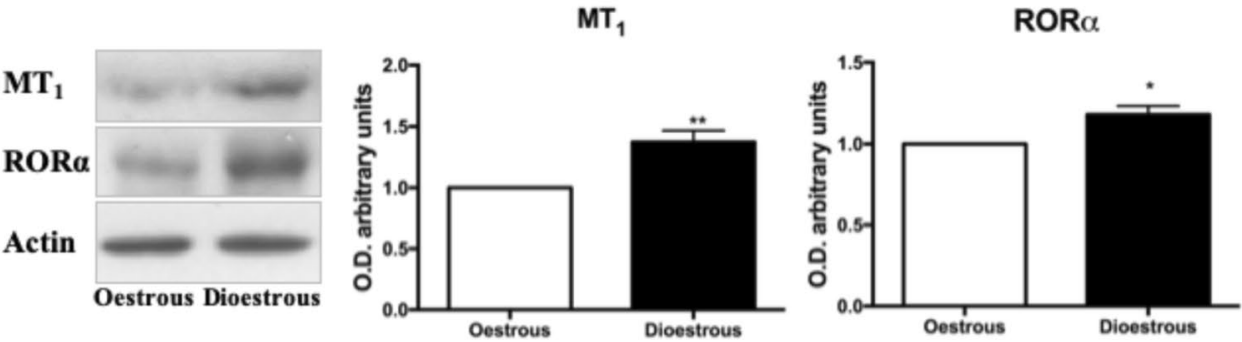

$\mathbf{b}$

Nrf2
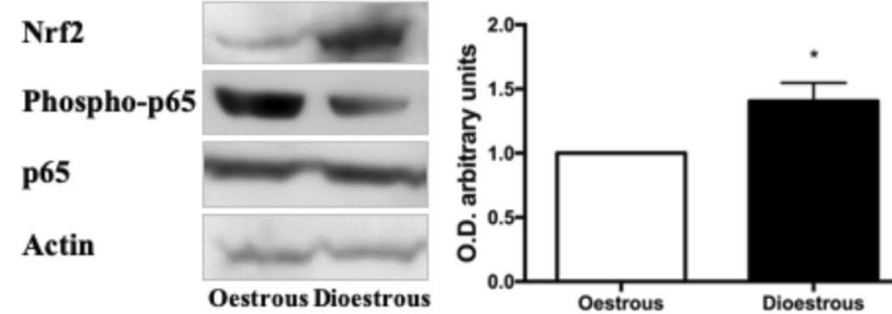

Ratio phospho-p65/p65

c

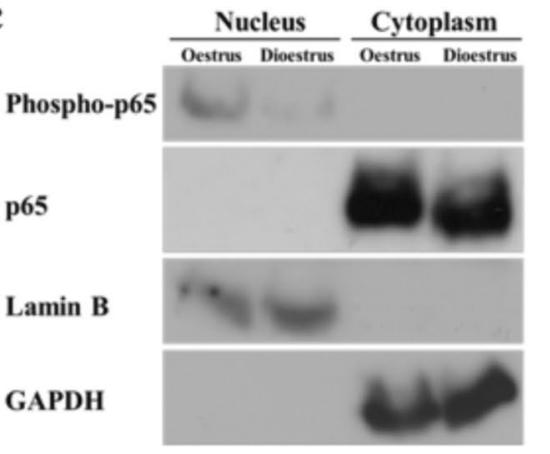

e

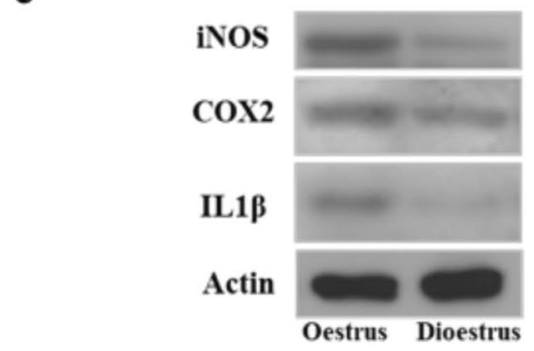

iNOS

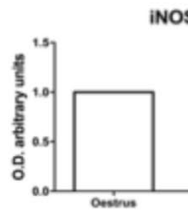

os

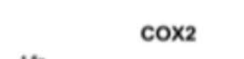

d
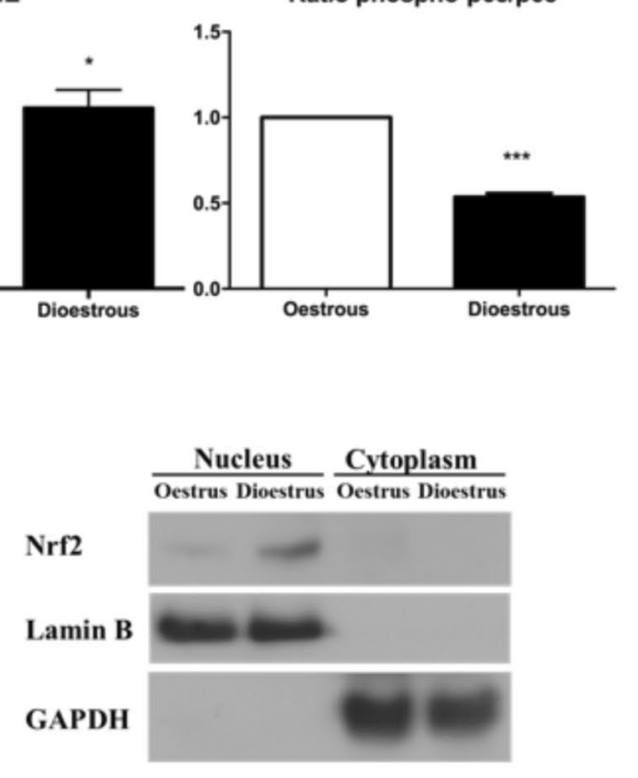

f

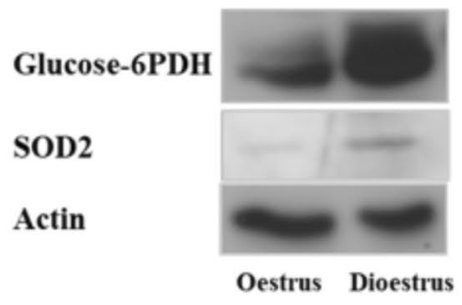

IL1 $\beta$

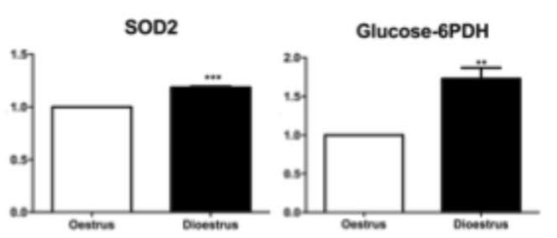

Figure 1. Melatonin role in the cellular response to oxidative stress. (a) MT-1, ROR $\alpha$ and (b) Nrf2, phospo-p65 and 655 protein band intensities in the different fractions of the Harderian glands from the oestrus and dioestrus phases. The bar graphs of MT-1 and ROR $\alpha(\mathbf{a})$ and Nrf2 (b) quantifies the optical densities of the western blot bands normalized to $ß$-actin and the phospho-p65/p65 ratio (b) as NF- $\kappa$ B activation marker. (c) Phopho-p65, p65 and (d) Nrf2, Lamin B and GAPDH protein band in nuclear and cytosolic fractions from the oestrus and dioestrus phases. (e) iNOS, COX2, IL1 $\beta$ and (f) Glucose-6PDH and SOD2 protein band intensities in the different fractions of the Harderian glands from the oestrus and dioestrus phases. The bar graphs of iNOS, COX2, IL1 $\beta$ (e) and Glucose-6PDH and SOD2 (f) quantifies the optical densities of the western blot bands normalized to $\beta$-actin. Data are expressed as the means \pm SEM and are calculated from at least three separate experiments. $*(\mathrm{p}<0.05), * *(\mathrm{p}<0.01), * * *(\mathrm{p}<0.001)$. 
$\mathbf{a}$

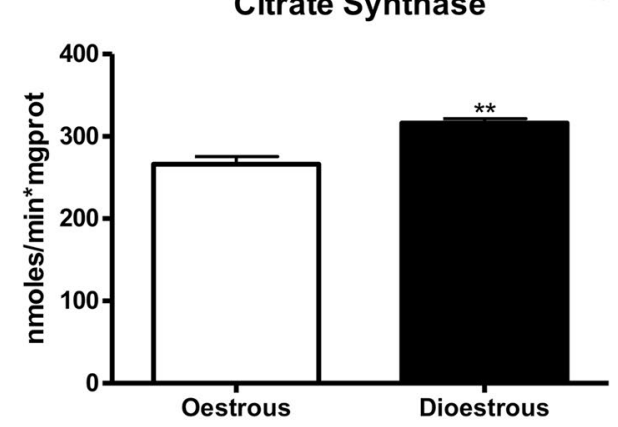

b

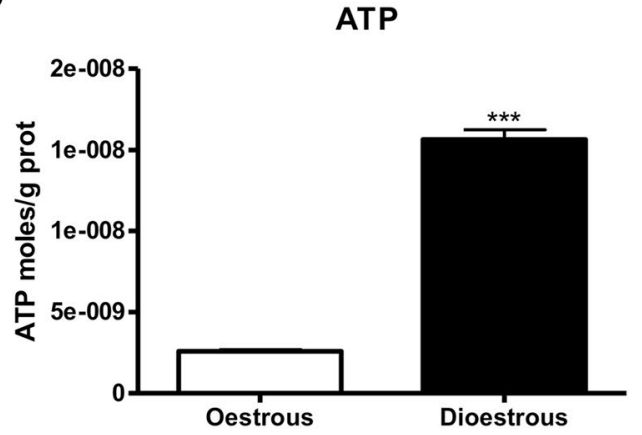

NIX

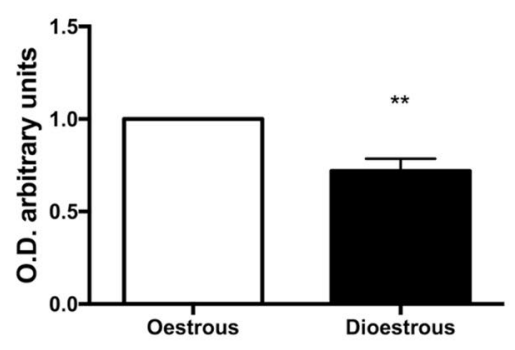

d
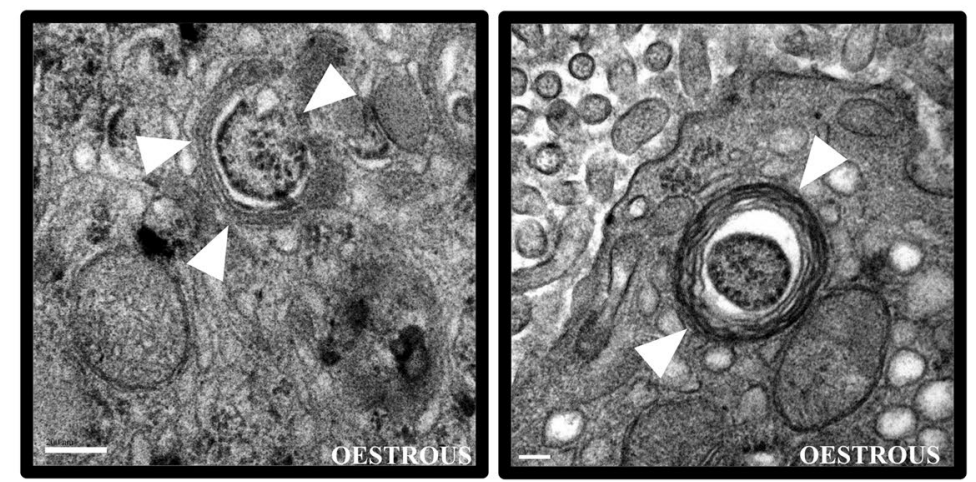

Figure 2. Mitochondrial status and clearance. Mitocondrial (a) Comparison of Citrate Synthase (CS) activity, which results are expressed in nmoles/min*mg protein. (b) ATP intracellular content, which results are expressed as ATP moles/g protein. (c) NIX protein band intensity and NIX bar graph that quantifies the optical densities of the western blot bands normalized to $B$-actin. Data are expressed as the means \pm SEM and are calculated from at least three separate experiments. $*(\mathrm{p}<0.05), * *(\mathrm{p}<0.01), * * *(\mathrm{p}<0.001)$. (d) Electron micrographs of cells in oestrus phase showing mitochondria within an autophagosome (arrowhead) as representative images. Similar results were obtained from three separate experiments. Scale bar, $0.2 \mu \mathrm{m}$.

described SQSTM1/p62 as a key mediator in lipolysis ${ }^{37}$ and also, in lipophagy ${ }^{38,39}$. SQSTM1/p62 protein (Fig. 3a, $\mathrm{p}<0.01$ ) and RNA (Fig. 3b, $\mathrm{p}<0.01$ ) levels were significantly lower during the dioestrus.

Lipolytic processes were studied by assaying the expression of lysosomal acid lipase (LAL) and the triglyceride (TG) levels. LAL is involved in the degradation of cholesteryl esters and triglycerides ${ }^{40}$. Our data showed LAL lower expression in the dioestrus phase (Fig. $3 \mathrm{c}, \mathrm{p}<0.05$ ). Concomitantly, TGs levels were also lower in the dioestrus phase (Fig. 3d, p < 0.001). LAL degrades the lipids provided by autophagy machinery (lipophagy) in the lysosome ${ }^{41}$. To characterize the level of lipophagy, we quantified the presence of the autophagy machinery components in the LD in both phases. First, we isolated LDs from HGs at oestrus and dioestrus, the expression of cytosolic lipases (ATGL and HSL) was assessed to evaluate classical lipolysis, no changes were found in the LD isolations. Then, we determined the presence and expression levels of autophagic proteins as Lamp1, Beclin, LC3-II, SQSTM1/p62, Plin2, Plin3 and, also, GAPDH to exclude cytosolic contamination ${ }^{42}$, by western blot. The expression of all the autophagic proteins was lower in LDs from the dioestrus phase while the total homogenate (Hom) was unchanged (Fig. 3e). This result indicates lower LD degradation in dioestrus than oestrus phase. Furthermore, our results from EM analysis of HGs during the oestrus cycle revealed the presence of lipid droplets inside the autophagosomes showing the high levels of lipid degradation (Fig. 3f). In order to further quantify the abundance of LDs we analyse the presence of the perilipin (Plin) family proteins that coats $\mathrm{LDs}^{43}$. As expected, we observed lower expression of Plin2 and Plin3 in dioestrus, indicating fewer LDs in this phase (Fig. 3e). 
$\mathbf{a}$

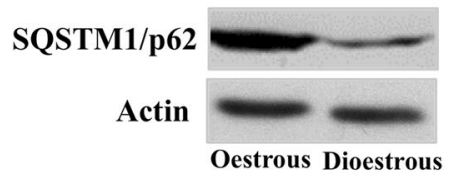

C

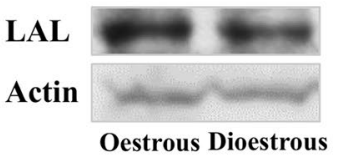

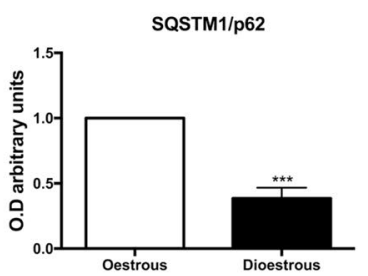

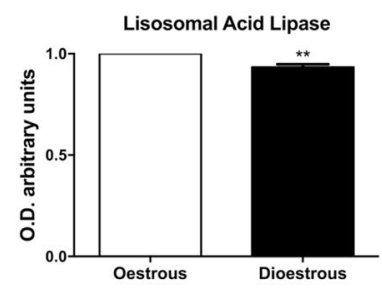

b

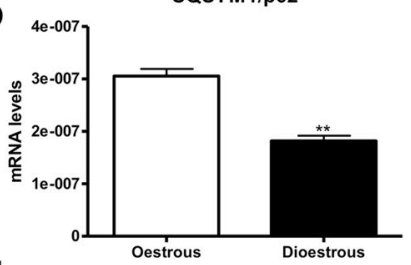

d

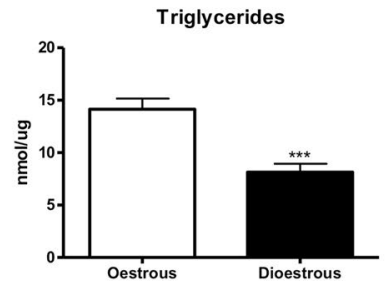

e
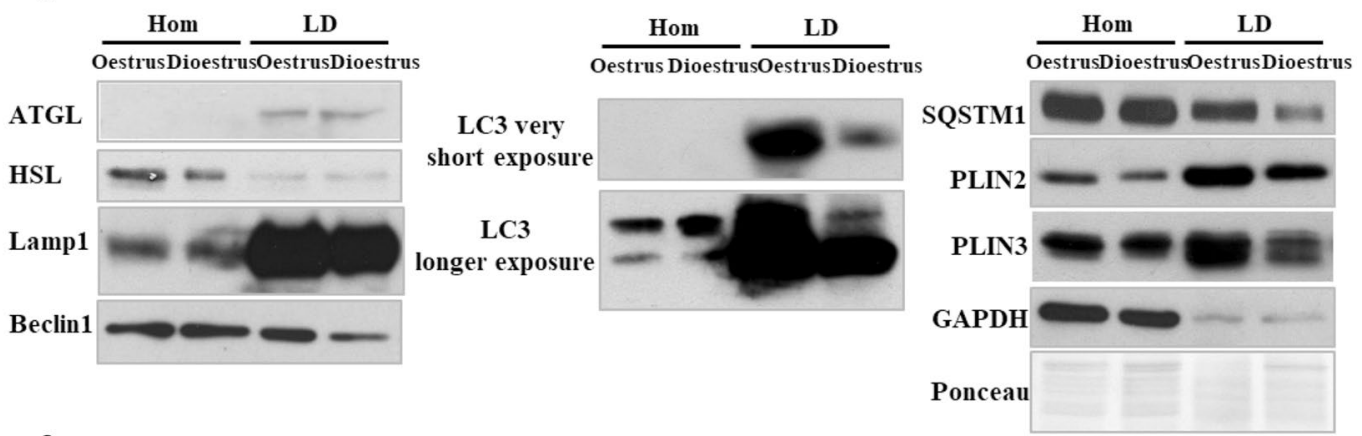

f
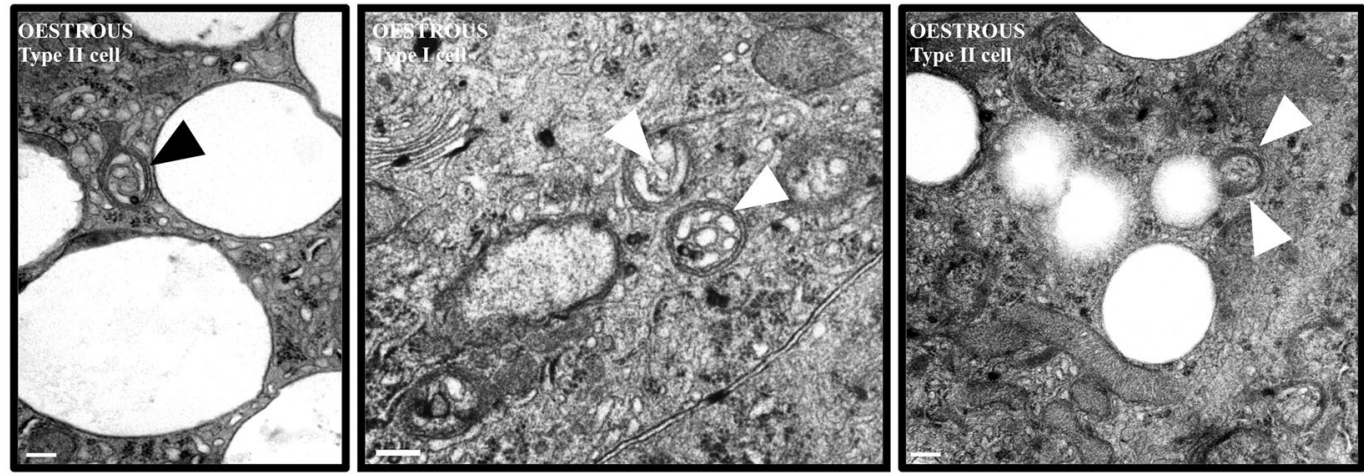

Figure 3. Lipolisis-related autophagy activity (a) SQSTM1/p62 and (c) LAL protein band intensities and its respective bar graphs that quantifies the optical densities of the western blot (WB) bands normalized to $B$-actin. (b) SQSTM1/p62 mRNA expression quantification, normalized to 18s. (d) Triglyceride levels in oestrus and dioestrus, results are expressed in nmoles/ $\mu \mathrm{g}$ protein. (e) WB for indicated proteins in total homogenates (Hom) and lipid droplets (LD). Data are expressed as the means \pm SEM and are calculated from at least three separate experiments, with each experiment performed in triplicate. $*(\mathrm{p}<0.05) * *(\mathrm{p}<0.01)(\mathbf{f})$ Electron micrographs of cells in oestrus phase showing lipid droplets within autophagosomes (arrowheads). Scale bar, $200 \mathrm{~nm}$.

We then use immunohistochemistry to confirm the previous results and determine whether LC3 (Fig. 4a) and Lamp1 (Fig. 4b), autophagosome and lysosomal markers respectively, co-localize with BODIPY, a dye that stains neutral lipids in the LD. We found less colocalization (through both Manders and Pearson tests) of both markers with the LDs in dioestrus phase (Fig. 4a,b, p $<0.001$ ), supporting the idea of higher lipophagy levels during oestrus.

\section{Discusion}

How sexual hormones modulate selective autophagy is poorly understood, this lack in knowledge aggravates the sexual bias we could easily find in biological research. Harderian gland (HG) has an intrinsic plasticity to quickly respond to several internal and external stimuli via both morphological and biochemical changes ${ }^{44}$. We have previously shown that female HG variations are dependent on the fluctuations in sex hormones during the oestrous 
$\mathbf{a}$

\section{Oestrus}

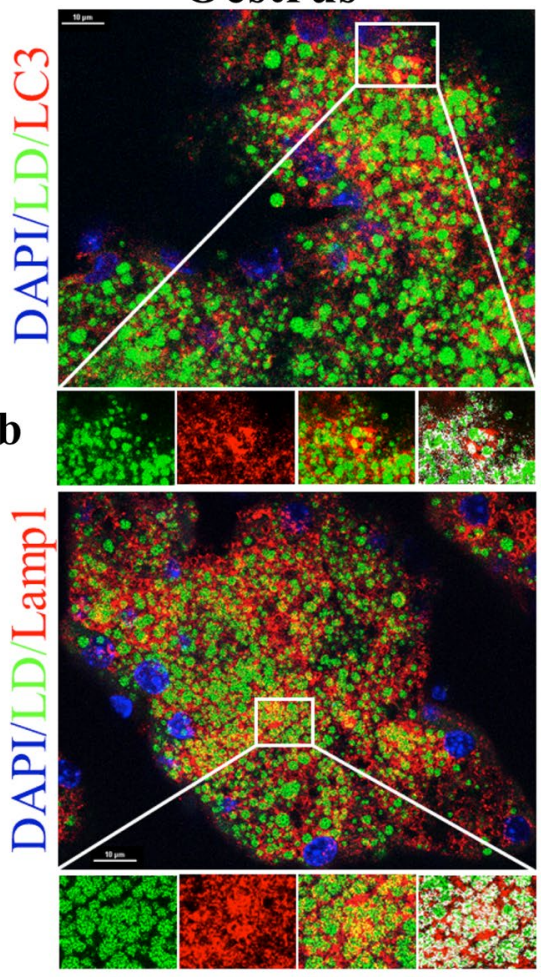

Dioestrus
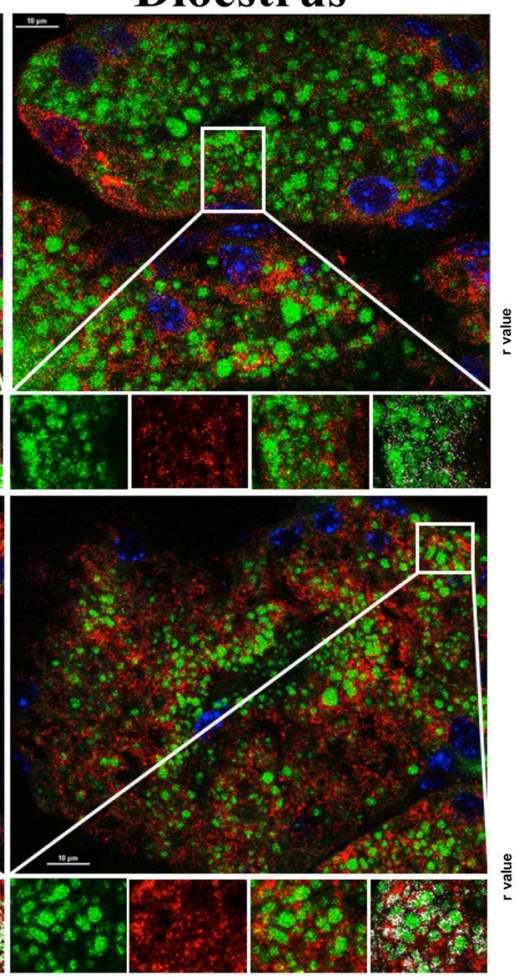
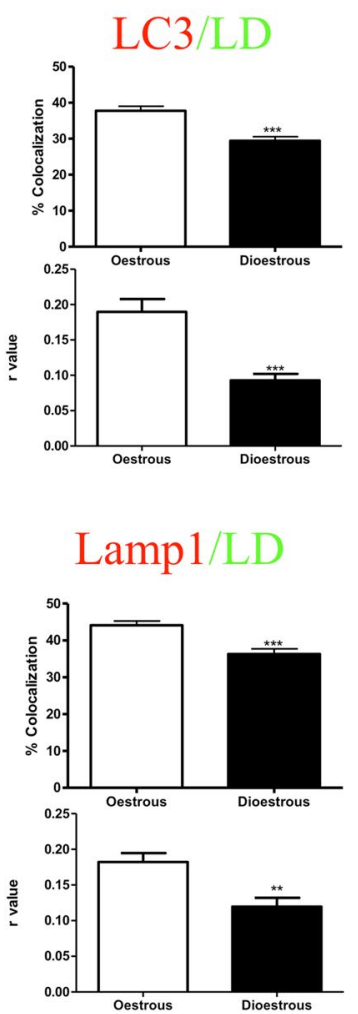

Figure 4. Differential lipophagy activation during the estrous cycle. Immunofluorescence for LC3 and BODIPY 493/503 (a) and Lamp1 and BODIPY 493/503. Colocalization determined by the "colocalization finder" plugin in Image is shown in white in the fourth enlarged panel. Enlarged image scale bar: $10 \mu \mathrm{m}$. (b) and its respective bar graphs that quantifies the percentage of colocalization.

cycle, which makes HG the perfect model to understand how these hormonal changes modulate autophagy. These autophagic changes during oestrous cycle are conserved among rodents $s^{45}$.

Melatonin is a hormone secreted by the pineal gland, usually related to protection against oxidative stress due to its antioxidant properties ${ }^{23}$. The rate of melatonin secretion varies synchronously with the vaginal cycle, being higher when the oestrogen levels are lower at dioestrus ${ }^{24}$. It has been described that melatonin displays an anti-estrogenic effect ${ }^{25}$ mediated by the membrane-bound receptor $\mathrm{MT}_{1}{ }^{46}$, although the contribution of nuclear receptors is still unclear ${ }^{46}$. Accordingly, melatonin receptors, both $\mathrm{MT}_{1}$ (plasma membrane) and ROR $\alpha$ (nucleus), are more abundant in dioestrus. Thus, the increased melatonin signalling, via its receptors, may be involved in reduction of pro-inflammatory mediators and alleviating the higher oxidative stress levels that exist during the oestrus phase ${ }^{12}$. In its role as antioxidant, melatonin has been described as inductor of Nrf2 activation, enhancing its nuclear translocation and subsequent antioxidant response (ARE) binding ${ }^{19,47}$. This translocation process has been observed in our study under dioestrus phase and an increase in antioxidant protein expression is also seen in dioestrus phase, what leads us to assume that melatonin antioxidant effect in the HG is mediated by its receptors and potentiated by Nrf2 activation in this phase along with minimal oestrogen levels.

The transcription factor NF- $\kappa \mathrm{B}$ is a pro-inflammatory factor activated by oxidative stress and inhibited by melatonin ${ }^{19}$. Here we have shown that NF- $\kappa B$ is activated during oestrus phase along with high expression of pro inflammatory factors (COX2 and IL1 $\beta$ ) and elevated levels of oxidative stress. Thus, activation of NF- $\kappa B$ favour the pro-inflammatory profile in $\mathrm{HG}$ at the oestrus phase. It has been also shown that NF- $\mathrm{BB}$ promotes mitophagy by SQSTM1/p62 induction ${ }^{48}$ and our analysis corroborates this role (Fig. S1). Accordingly, in our results female HG showed activation of SQSTM1/p62 in oestrus phase. Moreover, regarding mitochondrial clearance, the NIX protein, which is localized in the mitochondrial outer membrane, has been defined as a mitochondrial receptor for mitophagy ${ }^{35}$. Our present results showed a higher NIX expression in the female HG at the oestrus phase than in the dioestrus one. Additionally, we have observed the presence of mitophagosomes by EM (Fig. 2d). All together these data show the activation of mitophagy in the female's HG during oestrus mediated by NF- $\kappa \mathrm{B}$ activation. Moreover, mitochondrial depolarization, tested with Citrate synthase activity, and the subsequent mitochondrial ROS generation have been previously linked to NIX ability to directly activate the autophagy machinery via mTOR inhibition ${ }^{49}$. Consistent with these markers and data, mitochondrial energetic functions, assayed by ATP concentration, were significantly lower in the HGs during the oestrus phase. Therefore, increased levels of the mitophagy marker NIX, formation of mitophagosomes, and impaired mitochondrial functionality suggest an intense mitochondrial removal by selective autophagy in the female HG at the estrus phase promoted by NF- $\kappa$ B activation. 


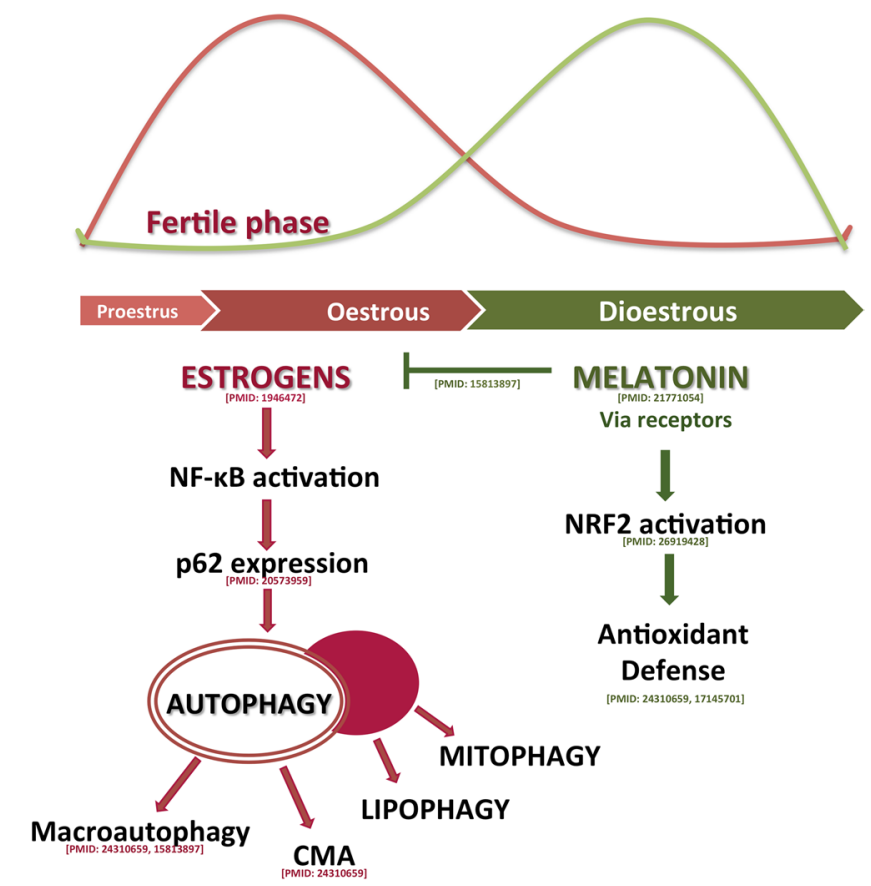

Figure 5. Modulation by melatonin of the estrous cycle mechanisms. The scheme proposes that oxidative stress is one of the key processes involved in the estrous cycle with melatonin as modulator of it.

The role of autophagy in lipid metabolism has been recently discovered and lipophagy has been shown as the main mechanism to mobilize lipid store ${ }^{15}$. Lipophagy was first discovered in liver and, currently, is described in many other tissues ${ }^{14}$. SQSTM1/p62 participates in the selective removal of organelles, as mitochondria or LDs through autophagy ${ }^{38,39}$. SQSTM1/p62 and other autophagic machinery directly contributes to the mobilization of lipids from LDs to lysosomes, where they get degraded by lipases ${ }^{14}$. Our data showed higher lipolytic activity in oestrus phase due to the higher expression of lysosomal acid lipase (LAL). Accordingly, autophagy and lysosomal proteins, but not cytosolic lipases, were accumulated in the LDs in this phase, showing higher lipophagy activity. Furthermore, our EM studies revealed the presence of LDs within the autophagosomes. Perilipins showed less expression in dioestrus, indicating lower number of LDs. Thus, our data relate lipolytic and lipophagy activities with the cellular changes usually observed in the HG during the oestrous cycle and, until now, not well understood. The higher lipophagy activity in oestrus could be the process behind the Type II cells disappearance.

We have shown that the HG in the oestrous cycle is characterized by important variations in oestrogen levels and oxidative stress (Fig. 5), where melatonin may be its primary moderator, in basis of its clear relationship with both factors: it is a well-known antioxidant and has important antiestrogenic effects. Melatonin interacts with 2 of the oestrogen-signalling pathways and it decreases circulating levels of oestradiol ${ }^{50,51}$. Melatonin levels are reduced in the oestrus phase and entail the activation of NF- $\kappa B$ and the elevated oxidative stress, which induces not only macroautophagy and $\mathrm{CMA}^{12,52,53}$ but also increases other selective autophagic processes. All associated to a decrease in mitochondrial activity, leading to degradation via mitophagy. Likewise, activation of NF- $\kappa B$ may also lead to lipolytic processes, as suggested by the increase of the autophagic and lysosomal proteins in the LDs and the degradation of large lipid droplets in Type II cells. These degradation processes require a high lysosomal effort, as shown by the increases in LAL expression. Melatonin, via both membrane and nuclear receptors, reduces pro-inflammatory mediators and enhances the expression of Nrf-2 in dioestrus phase. Consequently, autophagy is blocked, and porphyrin release is reduced, and the gland returns to a rest period.

\section{Materials and Methods}

Animals. Eight-week-old female Syrian hamsters (Mesocricetus auratus) (Harlan Interfauna Ibérica, Barcelona, Spain) were housed 2 per cage under long days with a 14:10 light:dark cycle (lights on daily from 07:00 to $21: 00)$ at $22 \pm 2{ }^{\circ} \mathrm{C}(\mathrm{n}=8$ per experimental condition). Hamsters received water and a standard pellet diet $a d$ libitum. The Oviedo University Local Animal Care and Use Committee approved the experimental protocols (reference 33443591). All experiments were carried out according to the Spanish Government Guide and the European Community Guide for Animal Care (Council Directive 86/609/EEC).

All animals were monitored daily by vaginal smears to determine their reproductive phase (proestrus, oestrus, metoestrus, dioestrus) according to the method of Orsini ${ }^{54}$, during 3 consecutive cycles. For studying opposite phases, we collected females during the oestrus and dioestrus phases because these phases present the highest plasmatic differences in oestrogen concentration during the oestrous cycle ${ }^{55}$. After determination of the specific oestrous phase, animals were sacrificed, and the hamster Harderian glands (HGs) were immediately removed, frozen in liquid nitrogen, and stored at $-80^{\circ} \mathrm{C}$ until performing of the experiments, HGs were used directly for lipid droplet isolation. 
Isolation of proteins. HGs $(0.1 \mathrm{~g})$ were homogenized using a Polytron homogenizer at $4{ }^{\circ} \mathrm{C}$ in $1 \mathrm{ml}$ of lysis buffer $(50 \mathrm{mM}$ Tris/ $\mathrm{HCl}, 150 \mathrm{mM} \mathrm{NaCl}$ at $\mathrm{pH} 7.4$ and protease and phosphatase inhibitors). The tissue homogenates were then centrifuged for $6 \mathrm{~min}$ at $3000 \mathrm{rpm}$ at $4{ }^{\circ} \mathrm{C}$. The supernatants were collected and centrifuged again under the same conditions. Nuclear and cytoplasmic fractions were prepared using the sucrose gradient $\operatorname{method}^{4}$. The protein concentration of the supernatants was measured by the method of Bradford ${ }^{56}$.

Isolation of lipid droplet (LD) fractions. LD fraction from Harderian gland was isolated following the protocol for brown adipose tissue with few alterations ${ }^{42}$. Tissues were homogenized in $0.25 \mathrm{M}$ sucrose and centrifuged at $6,800 \mathrm{~g}$ for $5 \mathrm{~min} / 4^{\circ} \mathrm{C}$. Supernatants including the fatty layer were centrifuged at $17,000 \mathrm{~g} / 10 \mathrm{~min} / 4^{\circ} \mathrm{C}$ to eliminate unwanted cellular fractions. Supernatant from the $17,000 \mathrm{~g}$ spin was adjusted to $20 \%$ sucrose and centrifuged in a discontinuous sucrose density gradient at $27,000 \mathrm{~g}$ for $30 \mathrm{~min}$ at $4{ }^{\circ} \mathrm{C}$. $\mathrm{LD}$ fractions were delipidated using successive washes in acetone and ether and solubilized in $2 \%$ SDS for immunoblotting.

Immunoblotting. Protein samples were prepared in western-blotting sample buffer $(65.8 \mathrm{mM}$ Tris- $\mathrm{HCl}, \mathrm{pH} 6.8$, $2.1 \%$ SDS, $26.3 \%(\mathrm{w} / \mathrm{v})$ glycerol, $0.01 \%$ Bromophenol Blue). SDS-polyacrylamide gels were run and analysed as previously described ${ }^{23,34}$. Primary antibodies applied were as follows: nuclear factor erythroid 2-related factor 2 (Nrf2), superoxide dismutase 2 (SOD2), retinoid-related orphan receptor alpha (ROR $\alpha)$ and melatonin receptor 1A (MT1) from Santa Cruz Biotechnology (Santa Cruz, CA, USA), adipose triglyceride lipase (ATGL), beclin 1, cyclooxygenase-2 (COX2), interleukin $1 \beta$ (IL1 $\beta$ ), hormone-sensitive lipase (HSL), lamin B, microtubule-associated protein 1A/1B-light chain 3 (LC3), nuclear factor kappa-B1 p50 subunit (NF- $\kappa B 1$ p50), nuclear factor kappa-B p65 subunit (NF- $\kappa B$ p65), nuclear factor kappa-B p65 subunit phosphorylated at Serine 536 (Phospho-NF- $\kappa B$ p65 [Ser536]) and sequestosome-1 (SQSTM1/p62) from Cell Signaling Technology (Boston, MA, USA), glyceraldehyde 3-phosphate dehydrogenase (GAPDH) and lysosomal acid lipase (LAL), inducible nitrate synthase (iNOS) and succinate dehydrogenase B (SDHB) antibody from Abcam (Cambridge, UK), lysosomal-associated membrane protein 1 (Lamp1) from Developmental Studies Hybridoma Bank (Iowa City, Iowa, USA); Bcl2/Adenovirus E1B 19kDa and protein-interacting protein 3-like (BNIP3L/NIX) and glucose 6-phosphate dehydrogenase (Glucose-6PDH) from Sigma-Aldrich (St. Louis, MO, USA), perilipin (PLIN) 2 (Progen Biotechnik, Heidelberg, DE) and PLIN3 (ProSci Inc, Poway, CA, US). Primary antibodies were mostly diluted 1:1000 in blocking buffer, except for Phospho-NF- $k B$ p65 (Ser536), which was diluted 1:500, and Nrf2, which was diluted 1:250. Goat $\beta$-actin antibody (Santa Cruz Biotechnology, Inc.) diluted at 1:1000 was always assayed as a loading reference. All the primary antibodies have been previously validated ${ }^{4,12,23,57}$. After washing in TBS-T (20 mM Tris-HCl, $150 \mathrm{mM} \mathrm{NaCl}, \mathrm{pH} 7.4$ and $0.05 \%$ Tween-20), the membranes were then incubated with the corresponding horseradish peroxidase-conjugated secondary antibody: anti-rabbit for most primaries, but anti-mouse for $\beta$-actin, or anti-rat for Lamp1 or anti-guinea pig for the PLIN2 (Santa Cruz Biotechnology, Inc.) diluted 1:2500. Binding of antibodies to their antigens was detected using the Western Blotting Luminol Reagent (sc-2048; Santa Cruz Biotechnology, Inc.) according to the manufacturer's protocol. Negative controls were performed with either no primary or no secondary antibodies. No bands were detected in any case.

The results were calculated from at least three separate experiments for each antibody and were normalized to actin. Band intensity was quantified using the Quantity One 1D analysis software v. 5.5.1. (Bio-Rad Laboratories Inc., Hercules, CA, USA). Then dioestrus values were normalized to oestrus values in order to make comparisons.

All the original blots are shown in the Supplementary Fig. 2.

Fluorescence microscopy. HGs were fixed overnight in $4 \%$ paraformaldehyde (PFA) solution at $4{ }^{\circ} \mathrm{C}$. After three washes in PBS, HGs were cryoprotected first overnight at $4^{\circ} \mathrm{C}$ in $15 \%$ sucrose and then 4 hours at room temperature in $30 \%$ sucrose. HGs were oriented and embedded in OCT and then frozen in liquid nitrogen. $10 \mu \mathrm{m}$ sections were obtained in a cryostat. Sections were rinse in PBS and then blocked with $3 \%$ horse serum, $1 \%$ BSA in $1 x$ PBS containing $0.4 \%$ triton X-100 for 1 hour at room temperature, then they were incubated with primary were incubated with primary antibodies Lamp1 (Developmental Studies Hybridoma Bank, Iowa City, Iowa, USA) and LC3 (Cell Signalling Technology, Boston, MA, USA) and secondary antibodies anti-rat and anti-rabbit, respectively (Alexa Fluor 647 conjugated, Invitrogen (Carlsbad, Ca, USA)). For lipid droplet (LD) detection, sections were incubated with BODIPY 493/503 for $20 \mathrm{~min}$ at RT (Molecular Probes Inc., Eugene, OR, USA). Mounting medium contained DAPI (4',6-diamidino-2-phenylindole) to visualize the nucleus (Molecular Probes Inc., Eugene, OR, USA). Negative controls were performed with either no primary or no secondary antibodies. No staining was detected in any case. Images were acquired on a Nikon A1R confocal inverted (Nikon Instruments INC., NY, USA) using X100 objective/1.4 numerical aperture. Images were acquired at similar exposure times in the same imaging session. Image slices of $0.2 \mu \mathrm{m}$ thickness were acquired and deconvolved using the Huygens (Huygens Essential, Hilversum, The Netherlands) acquisition/analysis software. Quantification was performed in deconvolved images after appropriate thresholding using the ImageJ software $(\mathrm{NIH})^{58}$ in a minimum of 30 acini from at least 3 experiments. Cellular fluorescence intensity was expressed as mean integrated density as a function of individual cell size. Percentage colocalization was calculated using the JACoP plugin in single Z-stack sections of deconvolved images (Manders and Pearson test). Colocalization is shown in native images and/or as white pixels using the "colocalization finder" plugin in ImageJ, using same threshold for all the images ${ }^{58}$.

Reverse transcription (RT). Total RNA was extracted using the Tripure ${ }^{\mathrm{TM}}$ Isolation Reagent (Roche Applied Science, Mannheim, Germany), according to the manufacturer's instructions. The yield of total RNA was determined by measuring the absorbance $(260 / 280 \mathrm{~nm})$ using a NanoDropND-1000 spectrophotometer (Nano-Drop Technologies, USA). RT was completed with the high-capacity cDNA Reverse Transcription Kit (Applied Biosystems, Foster City, CA, USA), following manufacturer instructions. Reactions were performed for $10 \mathrm{~min}$ at $25^{\circ} \mathrm{C}, 2 \mathrm{~h}$ at $37^{\circ} \mathrm{C}$ and terminated by heating for $5 \mathrm{sec}$ at $85^{\circ} \mathrm{C}$. The reaction mixture was maintained at $-20^{\circ} \mathrm{C}$ until further use. 


\begin{tabular}{|l|l|l|l|}
\hline Target gene & Forward primer $\left(\mathbf{5}^{\prime} \mathbf{3}^{\prime} \mathbf{)}\right.$ & Reverse primer $\left(\mathbf{5}^{\prime} \mathbf{3}^{\prime} \mathbf{)}\right.$ & Genbank \\
\hline 18S & GATTAAGTGCCCTTTGTA & GATCCGAGGGCCTCACTAAAC & v01270 \\
\hline p62 (Sequestosome 1) & CCATGGGTTTCTCGGATGAA & ATCCAGTATTCAAAGCACCCTCC & NM_175843.3 \\
\hline
\end{tabular}

Table 1. Real time PCR primers the GenBank accession numbers.

Quantitative real-time PCR. Quantitative real-time PCR of the different mRNAs was performed in triplicate using gene-specific primers and $\mathrm{SYBR}^{\circledR}$ Green. Oligonucleotide primers were designed using Primer Express 2.0 software (Applied Biosystems, Foster City, CA, USA). The primer sequences and corresponding GenBank accession numbers are given in Table 1 . As an internal control for normalization, PCR reactions were performed concurrently with the amplification of a reference gene, $18 \mathrm{~S}$ ribosomal RNA (rRNA) that proved to be stable in all the conditions studied.

Real time-PCR was performed on an Step-one plus (Applied Biosystems, Foster City, CA, USA) real-time thermal cycler using the SYBR ${ }^{\circledR}$ Green PCR Master Mix kit (Applied Biosystems, Foster City, CA, USA) with the following thermal cycler settings: one cycle of $10 \mathrm{~min}$ at $95^{\circ} \mathrm{C}, 40$ cycles of $15 \mathrm{~s}$ at $95^{\circ} \mathrm{C}$ and $1 \mathrm{~min}$ at $60^{\circ} \mathrm{C}$. Cycle thresholds for both genes were selected immediately above the baseline and within the linear range on log scaling. Each reaction $(20 \mu \mathrm{l})$ consisted of a $2 \mu \mathrm{lcDNA}$ aliquot, $300 \mathrm{nM}$ of each primer and $10 \mu \mathrm{l}$ of SYBR ${ }^{\circledR}$ Green PCR Master Mix containing AmpliTaq gold DNA polymerase. Increases in the amount of SYBR ${ }^{\circledR}$ Green reporter dye fluorescence during the amplification process were analysed with Sequence Detector software (SDS version 1.6 Applied Biosystems, Foster City, CA, USA). Relative change in expression of the target genes was determined by the following equation:

$$
\text { Fold change }=2-\Delta \mathrm{Ct}, \Delta \mathrm{Ct}=(\mathrm{Ct} \text { target }-\mathrm{Ct} 18 \mathrm{~S} \text { ribosomal RNA (rRNA)). }
$$

The Ct value is the cycle number at which the fluorescence signal crosses the designated threshold ${ }^{59}$.

Morphological studies. For ultrastructural studies, HGs were treated as previously described ${ }^{4}$. HGs were lightly fixed by immersion in a solution containing $1.5 \%$ glutaraldehyde and $2.5 \%$ paraformaldehyde in $0.1 \mathrm{M}$ phosphate buffer (pH 7.4). Fixation was continued overnight at $4{ }^{\circ} \mathrm{C}$ using fresh fixing solutions. Tissues were then postfixed in $1 \%$ Osmium (OsO4) for 2 hours. After dehydration in a graded acetone series, the tissue fragments were embedded in the epoxy resin TAAB 812 , and $1 \mu \mathrm{m}$ semithin sections were stained with toluidine blue. Ultrathin sections were collected on copper grids, stained with uranyl acetate-lead citrate, and examined using a Zeiss EM-109 transmission electron microscope (Zeiss, Oberkochen Germany) operating at $80 \mathrm{kV}$.

Mitochondrial functionality. As an indicator of the mitochondrial population, citrate synthase (CS) activity was determined spectrophotometrically at $412 \mathrm{~nm}$ and $30^{\circ} \mathrm{C}$, as previously described ${ }^{34}$.

The intracellular ATP content in the HGs was measured with an Adenosine- $5^{\prime}$-triphosphate bioluminescent assay kit (FL-AA, Sigma Aldrich Inc.). Bioluminescent luciferase-luciferin reactions provide the basis of simple, rapid, and highly sensitive assays for $\mathrm{ATP}^{60}$. Samples $(100 \mu \mathrm{L})$ of tissue homogenates diluted 1:100 were mixed with $100 \mu \mathrm{L}$ of ATP assay mix dilution buffer FL-AAB (pH 7.8). Light production was then immediately measured by luminescence using a Luminometer Turner Designs TD-20/20 (Turner BioSystems Inc., Sunnyvale, CA, USA) ${ }^{61}$.

Triglyceride levels. The triglyceride (TG) levels were determined using a triglyceride quantification kit following the manufacturer's indications (Abcam, Cambridge, UK). In this assay, triglycerides were converted to free fatty acids and glycerol. The glycerol was oxidized and combined with a probe to generate the luminometric signal. HG gland samples were lysed using NP-40 solution, then incubated with the lipase for 20 minutes. A final incubation of 1 hour with the Triglyceride reaction to ensure the oxidation of the glycerol and the colorimetric reaction. Measurement was performed at OD $570 \mathrm{~nm}$ in a Varioskan Flash (Thermo Fisher, Waltham, MA, USA).

\section{Data availability}

The authors declare that the data supporting the findings of this study are available within the paper.

Received: 14 June 2019; Accepted: 7 November 2019;

Published online: 09 December 2019

\section{References}

1. Niel, L., Willemsen, K. R., Volante, S. N. \& Monks, D. A. Sexual dimorphism and androgen regulation of satellite cell population in differentiating rat levator ani muscle. Dev Neurobiol 68, 115-122, https://doi.org/10.1002/dneu.20580 (2008).

2. Kim, Y. et al. Brain-wide Maps Reveal Stereotyped Cell-Type-Based Cortical Architecture and Subcortical Sexual Dimorphism. Cell 171, 456-469 e422, https://doi.org/10.1016/j.cell.2017.09.020 (2017).

3. Du, L. et al. Starving neurons show sex difference in autophagy. J Biol Chem 284, 2383-2396, https://doi.org/10.1074/jbc. M804396200 (2009).

4. Vega-Naredo, I. et al. Sexual dimorphism of autophagy in Syrian hamster Harderian gland culminates in a holocrine secretion in female glands. Autophagy 5, 1004-1017, https://doi.org/10.4161/auto.5.7.9610 (2009).

5. Zielniok, K., Sobolewska, A. \& Gajewska, M. Mechanisms of autophagy induction by sex steroids in bovine mammary epithelial cells. J Mol Endocrinol 59, 29-48, https://doi.org/10.1530/JME-16-0247 (2017).

6. Zielniok, K., Motyl, T. \& Gajewska, M. Functional interactions between 17 beta -estradiol and progesterone regulate autophagy during acini formation by bovine mammary epithelial cells in 3D cultures. Biomed Res Int 2014, 382653, https://doi. org/10.1155/2014/382653 (2014). 
7. Coto-Montes, A. et al. Sexual autophagic differences in the androgen-dependent flank organ of Syrian hamsters. J Androl 30, 113-121, https://doi.org/10.2164/jandrol.108.005355 (2009).

8. Sakai, T. The mammalian Harderian gland: morphology, biochemistry, function and phylogeny. Arch Histol Jpn 44, 299-333 (1981).

9. Payne, A. P., McGadey, J., Moore, M. H. \& Thompson, G. G. Changes in Harderian gland activity in the female golden hamster during the oestrous cycle, pregnancy and lactation. The Biochemical journal 178, 597-604 (1979).

10. Hoffman, R. A., Johnson, L. B. \& Reiter, R. J. Harderian glands of golden hamsters: temporal and sexual differences in immunoreactive melatonin. J Pineal Res 2, 161-168 (1985).

11. Menendez-Pelaez, A., Lopez-Gonzalez, M. A. \& Guerrero, J. M. Melatonin binding sites in the Harderian gland of Syrian hamsters: sexual differences and effect of castration. J Pineal Res 14, 34-38 (1993).

12. Garcia-Macia, M. et al. Autophagic and proteolytic processes in the Harderian gland are modulated during the estrous cycle. Histochem Cell Biol 141, 519-529, https://doi.org/10.1007/s00418-013-1170-1 (2014).

13. Coto-Montes, A. et al. Analysis of constant tissue remodeling in Syrian hamster Harderian gland: intra-tubular and inter-tubular syncytial masses. J Anat 222, 558-569, https://doi.org/10.1111/joa.12040 (2013).

14. Singh, R. \& Cuervo, A. M. Lipophagy: connecting autophagy and lipid metabolism. Int J Cell Biol 2012, 282041, https://doi. org/10.1155/2012/282041 (2012).

15. Singh, R. et al. Autophagy regulates lipid metabolism. Nature 458, 1131-1135, https://doi.org/10.1038/nature07976 (2009).

16. Pearson, G. L. et al. Lysosomal acid lipase and lipophagy are constitutive negative regulators of glucose-stimulated insulin secretion from pancreatic beta cells. Diabetologia 57, 129-139, https://doi.org/10.1007/s00125-013-3083-x (2014).

17. Duta-Mare, M. et al. Lysosomal acid lipase regulates fatty acid channeling in brown adipose tissue to maintain thermogenesis. Biochim Biophys Acta Mol Cell Biol Lipids 1863, 467-478, https://doi.org/10.1016/j.bbalip.2018.01.011 (2018).

18. Reiter, R. J. The pineal and its hormones in the control of reproduction in mammals. Endocr Rev 1, 109-131 (1980).

19. Negi, G., Kumar, A. \& Sharma, S. S. Melatonin modulates neuroinflammation and oxidative stress in experimental diabetic neuropathy: effects on NF-kappaB and Nrf2 cascades. J Pineal Res 50, 124-131, https://doi.org/10.1111/j.1600-079X.2010.00821.x (2011).

20. Coto-Montes, A. et al. Role of melatonin in the regulation of autophagy and mitophagy: a review. Molecular and cellular endocrinology 361, 12-23, https://doi.org/10.1016/j.mce.2012.04.009 (2012).

21. Tamarindo, G. H. et al. Melatonin and Docosahexaenoic Acid Decrease Proliferation of PNT1A Prostate Benign Cells via Modulation of Mitochondrial Bioenergetics and ROS Production. Oxid Med Cell Longev 2019, 5080798, https://doi. org $/ 10.1155 / 2019 / 5080798(2019)$.

22. Martin, M. et al. Melatonin-induced increased activity of the respiratory chain complexes I and IV can prevent mitochondrial damage induced by ruthenium red in vivo. J Pineal Res 28, 242-248 (2000).

23. Vega-Naredo, I. et al. Melatonin modulates autophagy through a redox-mediated action in female Syrian hamster Harderian gland controlling cell types and gland activity. J Pineal Res 52, 80-92, https://doi.org/10.1111/j.1600-079X.2011.00922.x (2012).

24. Ozaki, Y., Wurtman, R. J., Alonso, R. \& Lynch, H. J. Melatonin secretion decreases during the proestrous stage of the rat estrous cycle. Proc Natl Acad Sci USA 75, 531-534 (1978).

25. Rato, A. G. et al. Melatonin blocks the activation of estrogen receptor for DNA binding. FASEB J 13, 857-868 (1999).

26. Tomas-Zapico, C. et al. Coexpression of MT1 and RORalpha1 melatonin receptors in the Syrian hamster Harderian gland. J Pineal Res 39, 21-26, https://doi.org/10.1111/j.1600-079X.2005.00210.x (2005).

27. Caballero, B. et al. Favorable effects of a prolonged treatment with melatonin on the level of oxidative damage and neurodegeneration in senescence-accelerated mice. J Pineal Res 45, 302-311, https://doi.org/10.1111/j.1600-079X.2008.00591.x (2008).

28. Zhang, D. D. Mechanistic studies of the Nrf2-Keap1 signaling pathway. Drug Metab Rev 38, 769-789, https://doi. org/10.1080/03602530600971974 (2006).

29. Lau, A. et al. A noncanonical mechanism of Nrf2 activation by autophagy deficiency: direct interaction between Keap1 and p62. Mol Cell Biol 30, 3275-3285, https://doi.org/10.1128/MCB.00248-10 (2010).

30. Michiels, C., Minet, E., Mottet, D. \& Raes, M. Regulation of gene expression by oxygen: NF-kappaB and HIF-1, two extremes. Free radical biology \& medicine 33, 1231-1242 (2002).

31. Caballero, B., Veenman, L. \& Gavish, M. Role of mitochondrial translocator protein (18 kDa) on mitochondrial- related cell death processes. Recent patents on endocrine, metabolic \& immune drug discovery 7, 86-101 (2013).

32. Leon, J. et al. Melatonin and mitochondrial function. Life Sci 75, 765-790, https://doi.org/10.1016/j.lfs.2004.03.003 (2004).

33. Schatz, G. Mitochondria: beyond oxidative phosphorylation. Biochim Biophys Acta 1271, 123-126, https://doi.org/10.1016/09254439(95)00018-y (1995).

34. Garcia-Macia, M. et al. Melatonin induces neural SOD2 expression independent of the NF-kappaB pathway and improves the mitochondrial population and function in old mice. J Pineal Res 50, 54-63, https://doi.org/10.1111/j.1600-079X.2010.00809.x (2011).

35. Novak, I. et al. Nix is a selective autophagy receptor for mitochondrial clearance. EMBO Rep 11, 45-51, https://doi.org/10.1038/ embor.2009.256 (2010).

36. Lin, X. et al. Interaction Domains of p62: A Bridge Between p62 and Selective Autophagy. DNA and cell biology, https://doi. org/10.1089/dna.2012.1915 (2013).

37. Lee, S. J. et al. A functional role for the p62-ERK1 axis in the control of energy homeostasis and adipogenesis. EMBO Rep 11, 226-232, https://doi.org/10.1038/embor.2010.7 (2010).

38. Tatsumi, T. et al. Forced lipophagy reveals that lipid droplets are required for early embryonic development in mouse. Development 145, https://doi.org/10.1242/dev.161893 (2018).

39. Wang, L. et al. Ethanol-triggered Lipophagy Requires SQSTM1 in AML12 Hepatic Cells. Sci Rep 7, 12307, https://doi.org/10.1038/ s41598-017-12485-2 (2017).

40. Anderson, R. A., Bryson, G. M. \& Parks, J. S. Lysosomal acid lipase mutations that determine phenotype in Wolman and cholesterol ester storage disease. Molecular genetics and metabolism 68, 333-345, https://doi.org/10.1006/mgme.1999.2904 (1999).

41. Skop, V. et al. Autophagy-lysosomal pathway is involved in lipid degradation in rat liver. Physiol Res 61, 287-297, 932285 (2012).

42. Martinez-Lopez, N. et al. Autophagy in the CNS and Periphery Coordinate Lipophagy and Lipolysis in the Brown Adipose Tissue and Liver. Cell Metab 23, 113-127, https://doi.org/10.1016/j.cmet.2015.10.008 (2016).

43. Itabe, H., Yamaguchi, T., Nimura, S. \& Sasabe, N. Perilipins: a diversity of intracellular lipid droplet proteins. Lipids Health Dis 16, 83, https://doi.org/10.1186/s12944-017-0473-y (2017).

44. Rodriguez-Colunga, M. J. et al. Development and androgen regulation of the secretory cell types of the Syrian hamster (Mesocricetus auratus) Harderian gland. Cell and tissue research 274, 189-197 (1993).

45. Koenig, U. et al. Autophagy facilitates secretion and protects against degeneration of the Harderian gland. Autophagy 11, 298-313, https://doi.org/10.4161/15548627.2014.978221 (2015).

46. Girgert, R., Hanf, V., Emons, G. \& Grundker, C. Membrane-bound melatonin receptor MT1 down-regulates estrogen responsive genes in breast cancer cells. J Pineal Res 47, 23-31, https://doi.org/10.1111/j.1600-079X.2009.00684.x (2009).

47. Aparicio-Soto, M., Alarcon-de-la-Lastra, C., Cardeno, A., Sanchez-Fidalgo, S. \& Sanchez-Hidalgo, M. Melatonin modulates microsomal PGE synthase 1 and NF-E2-related factor-2-regulated antioxidant enzyme expression in LPS-induced murine peritoneal macrophages. British journal of pharmacology 171, 134-144, https://doi.org/10.1111/bph.12428 (2014). 
48. Zhong, Z. et al. NF-kappaB Restricts Inflammasome Activation via Elimination of Damaged Mitochondria. Cell 164, 896-910, https://doi.org/10.1016/j.cell.2015.12.057 (2016).

49. Ding, W. X. et al. Nix is critical to two distinct phases of mitophagy, reactive oxygen species-mediated autophagy induction and Parkin-ubiquitin-p62-mediated mitochondrial priming. J Biol Chem 285, 27879-27890, https://doi.org/10.1074/jbc.M110.119537 (2010).

50. Sanchez-Barcelo, E. J. et al. Melatonin-estrogen interactions in breast cancer. J Pineal Res 38, 217-222, https://doi.org/10.1111/ j.1600-079X.2004.00207.x (2005).

51. Gonzalez-Gonzalez, A., Mediavilla, M. D. \& Sanchez-Barcelo, E. J. Melatonin: A Molecule for Reducing Breast Cancer Risk. Molecules 23, https://doi.org/10.3390/molecules23020336 (2018).

52. Poole, M. C., Mahesh, V. B. \& Costoff, A. Morphometric analysis of the autophagic and crinophagic lysosomal systems in mammotropes throughout the estrous cycle of the rat. Cell Tissue Res 220, 131-137 (1981).

53. Riley, J. C. \& Behrman, H. R. Oxygen radicals and reactive oxygen species in reproduction. Proc Soc Exp Biol Med 198, 781-791 (1991).

54. Orsini, M. W. The external vaginal phenomena characterizing the stages of the estrous cycle, pregnancy, pseudopregnancy, lactation, and the anestrous hamster, Mesocricetus auratus waterhouse. Proceedings of the animal care panel 11, 193-206 (1961).

55. Lisk, R. D. In The hamster: reproduction and behavior 23-51 (New York: Plenum Press, 1985).

56. Bradford, M. M. A rapid and sensitive method for the quantitation of microgram quantities of protein utilizing the principle of protein-dye binding. Analytical biochemistry 72, 248-254 (1976).

57. Klionsky, D. J. et al. Guidelines for the use and interpretation of assays for monitoring autophagy (3rd edition). Autophagy 12, 1-222, https://doi.org/10.1080/15548627.2015.1100356 (2016).

58. Schneider, C. A., Rasband, W. S. \& Eliceiri, K. W. NIH Image to ImageJ: 25 years of image analysis. Nature methods $9,671-675$ (2012).

59. Livak, K. J. \& Schmittgen, T. D. Analysis of relative gene expression data using real-time quantitative PCR and the 2(-Delta Delta C(T)) Method. Methods 25, 402-408, https://doi.org/10.1006/meth.2001.1262 (2001).

60. Taylor, A. L. et al. Bioluminescence detection of ATP release mechanisms in epithelia. The American journal of physiology 275, C1391-1406, https://doi.org/10.1152/ajpcell.1998.275.5.C1391 (1998).

61. Yen, C. C. et al. The diabetogenic effects of the combination of humic acid and arsenic: in vitro and in vivo studies. Toxicology letters 172, 91-105, https://doi.org/10.1016/j.toxlet.2007.05.008 (2007).

\section{Acknowledgements}

We are members of the INPROTEOLYS, SEBBM, SEFAGIA and INEUROPA network. We acknowledge Fibrosis and Bolaños 'lab for their support during the manuscript revisions. This work was supported by FISS18-PI17/02009, C0120R3166, C0245R4032 and BH182173. MG-M has a postdoctoral fellowship from the Instituto de Salud Carlos III (ISCIII), Ministerio de Ciencia, Innovación y Universidades. Financial support from the University of Oviedo is also acknowledged.

\section{Author contributions}

M.G.-M., A.S.-L., B.C., A.R.-G., B.de.L.-D., Y.P., S.M.R.-G. performed experiments and analysed the data; M.G.-M. and A.S.-L. performed statistical analysis; M.G.-M., J.A.B. and A.C.-M. designed the study design and supervised the project; M.G.-M., A.S.-L. and A.C.-M. wrote the paper. All authors reviewed the manuscript.

\section{Competing interests}

The authors declare no competing interests.

\section{Additional information}

Supplementary information is available for this paper at https://doi.org/10.1038/s41598-019-54743-5.

Correspondence and requests for materials should be addressed to M.G.-M. or A.C.-M.

Reprints and permissions information is available at www.nature.com/reprints.

Publisher's note Springer Nature remains neutral with regard to jurisdictional claims in published maps and institutional affiliations.

Open Access This article is licensed under a Creative Commons Attribution 4.0 International License, which permits use, sharing, adaptation, distribution and reproduction in any medium or format, as long as you give appropriate credit to the original author(s) and the source, provide a link to the Creative Commons license, and indicate if changes were made. The images or other third party material in this article are included in the article's Creative Commons license, unless indicated otherwise in a credit line to the material. If material is not included in the article's Creative Commons license and your intended use is not permitted by statutory regulation or exceeds the permitted use, you will need to obtain permission directly from the copyright holder. To view a copy of this license, visit http://creativecommons.org/licenses/by/4.0/.

(C) The Author(s) 2019 